\title{
Evolution of the density self-correlation in developing Richtmyer-Meshkov turbulence
}

\author{
C. D. Tomkins $\dagger$, B. J. Balakumar, G. Orlicz, K. P. Prestridge \\ and J. R. Ristorcelli
}

Physics Division, Los Alamos National Laboratory, Los Alamos, NM 87545, USA

(Received 2 October 2012; revised 12 July 2013; accepted 12 August 2013; first published online 24 October 2013)

\begin{abstract}
Turbulent mixing in a Richtmyer-Meshkov unstable light-heavy-light (air-SF ${ }_{6}$-air) fluid layer subjected to a shock (Mach 1.20) and a reshock (Mach 1.14) is investigated using ensemble statistics obtained from simultaneous velocity-density measurements. The mixing is driven by an unstable array of initially symmetric vortices that induce rapid material mixing and create smaller-scale vortices. After reshock the flow appears to transition to a turbulent (likely three-dimensional) state, at which time our planar measurements are used to probe the developing flow field. The density selfcorrelation $b=-\langle\rho v\rangle$ (where $\rho$ and $v$ are the fluctuating density and specific volume, respectively) and terms in its evolution equation are directly measured experimentally for the first time. Amongst other things, it is found that production terms in the $b$ equation are balanced by the dissipation terms, suggesting a form of equilibrium in $b$. Simultaneous velocity measurements are used to probe the state of the incipient turbulence. A length-scale analysis suggests that an inertial range is beginning to form, consistent with the onset of a mixing transition. The developing turbulence is observed to reduce non-Boussinesq effects in the flow, which are found to be small over much of the layer after reshock. Second-order two-point structure functions of the density field exhibit a power-law behaviour with a steeper exponent than the standard $2 / 3$ power found in canonical turbulence. The absence of a significant $2 / 3$ region is observed to be consistent with the state of the flow, and the emergence of the steeper power-law region is discussed.
\end{abstract}

Key words: transition to turbulence, turbulence modelling, turbulent mixing

\section{Introduction}

High-resolution mean and fluctuating velocity and density field measurements are made in a Richtmyer-Meshkov (RM) flow to provide new insights about production and dissipation in a two-fluid, developing turbulent flow field. Understanding the nature of mixing in this shock-driven turbulent field is critical to modelling and simulation efforts with important consequences for many flows. In inertial confinement 
fusion reactions, the mixing induced by the RM instability (created by a converging shock wave on the fuel-shell interface) results in fuel contamination and reduced fusion yield (Lindl, McCrory \& Campbell 1992). RM instabilities increase the mixing between the fuel and the oxidizer in supersonic engines, enhancing combustion efficiency (Yang, Kubota \& Zukoski 1993). The patterns observed in supernova explosions and ejecta from shock-induced metal melt have also been attributed to RM instability (Arnett et al. 1987).

Variable-density (VD) mixing offers challenges beyond even those of the classical turbulent mixing problem. In VD mixing of fluids with vastly different densities, e.g. $\rho_{2} / \rho_{1}>3$ and for which $\rho / \bar{\rho} \geqslant 1$, there are several physical processes that do not appear in constant-density mixing, and new mixing physics is seen (Livescu \& Ristorcelli 2009). Here $\rho=\rho^{*}-\bar{\rho}$ denotes the fluctuation of the mixture density about its mean $\bar{\rho}$, with $\rho^{*}$ representing the total (instantaneous) mixture density. In this article we address binary VD mixing between two fluids with densities $\rho_{1}$ and $\rho_{2}$ and with an 'equation of state', once the shock has passed and the compressible fluctuations are small (the low turbulent Mach number limit), of the form

$$
\frac{1}{\rho^{*}}=\frac{Y_{1}}{\rho_{1}}+\frac{1-Y_{1}}{\rho_{2}} \text {. }
$$

With the density acutely dependent on the mixing mass fraction $Y_{1}$, the advection term in the Navier-Stokes equation has cubic nonlinearity associated with the differential acceleration of the different fluids, which produces density-dependent mixing effects (Livescu \& Ristorcelli 2009). In these flows the conservation equation for the mass fraction vector, $Y_{\alpha}$, is (Williams 1994)

$$
\left(\rho^{*} Y_{\alpha}\right),_{t}+\left(\rho^{*} Y_{\alpha} U_{k}\right),_{k}=-J_{k, k}^{\alpha}, \quad \alpha=1, \ldots, N,
$$

where $\rho^{*}, U_{k}$ and $J_{k}^{\alpha}$ are the mixture density, mixture velocity and diffusive mass fluxes, respectively. The problem described in terms of the mass fraction variables (1.2) is highly nonlinear due to: (i) $\rho^{*}=\rho^{*}(\boldsymbol{Y})$ in the advective term; and (ii) the diffusive flux, $J_{k}^{\alpha}$ (unlike the Boussinesq case with $\rho \approx$ constant) depends, importantly, on $\rho$ and thus on all components of $Y_{\alpha}$. The diffusive flux in a simple class of multicomponent VD mixing is

$$
J_{k}^{\alpha}=-\rho^{*}(\mathbf{Y}) \mathscr{D} Y_{\alpha, k},
$$

where the diffusion coefficient $\mathscr{D}$ is assumed constant and uniform for all species. For the present binary mixing case the diffusive flux of species 1 is then

$$
J_{k}^{1}=-\frac{\rho_{2}}{1+Y_{1}\left(\rho_{2} / \rho_{1}-1\right)} \mathscr{D} Y_{1, k} .
$$

In extreme cases, e.g. $\rho_{2} / \rho_{1}>10$, the diffusive flux varies considerably for various $0<Y_{1}<1$. Thus, the nonlinear nature of the diffusion process is clear. This leads to phenomena not seen in the $\rho \approx$ constant case that relate to the fact that heavy fluid mixing into light occurs qualitatively differently than light into heavy, introducing a new source of skewness of the probability density function (p.d.f.) (Livescu \& Ristorcelli 2008, 2009; Bakosi \& Ristorcelli 2011). Hence, VD mixing exhibits interesting new behaviours when extreme effects are considered, and represents a great predictive challenge in fluid mechanics even for more moderate VD effects (which is the case in the present flow).

As shown above, one fundamental aspect of the complexity of VD mixing is a dependence both on the velocity and density fields, where the density field actively 
modulates the response of the fluid to a given stress condition (called level-2 mixing by Dimotakis (2005)). This feedback between the velocity and density fields demands the application of a suitable diagnostic, one that can measure both of these quantities simultaneously, to begin to understand this type of turbulent mixing. Recent advances in the successful implementation of simultaneous particle-image velocimetry (PIV)-planar laser-induced fluorescence (PLIF) diagnostics to RM flows allow the measurement of instantaneous velocity and density two-dimensional fields at the same time (Balakumar et al. 2008). An earlier paper focused on fundamental velocity statistics and select density-velocity correlations relevant to mixing, including the streamwise mass flux $\left(\overline{\rho u_{1}}\right)$ and components of the general Reynolds stress tensor, $R_{i j}=\overline{\rho^{*} u_{i}^{\prime \prime} u_{j}^{\prime \prime}}$ (Balakumar et al. 2012). (Here the double prime denotes fluctuations from a density-weighted or Favre average.) The behaviour of the streamwise mass flux is quantified and discussed, and it is shown that the in-plane self-correlation Reynolds stresses $\left(R_{11}\right.$ and $\left.R_{22}\right)$ are dominant relative to the cross-correlation term $\left(R_{12}\right)$. Further, when the mass-weighted Reynolds stresses are written in terms of the standard (non-mass-weighted) fluctuations, one of the three terms, the mean density and cross-velocity product term $\bar{\rho} \overline{u_{i} u_{j}}$, is shown to be dominant in its contribution, and hence critical for accurate modelling.

In the present paper, we initially focus on the density self-correlation (DSC), or $b=-\langle\rho v\rangle$, an important quantity in mixing in VD flows due to its role in the mass flux equation, and a fundamental quantity in second-moment turbulence models. We experimentally measure the DSC and the terms in its evolution equation for the first time (§4). In $\S 5$, we perform a length-scale analysis to quantify the state of the developing turbulence and place the DSC results in the context of the nascent mixing transition. Several other aspects of the VD flow are examined and interpreted in later sections, including non-Boussinesq effects, the time required to achieve equilibrium, and the (non-classical) behaviour of density structure functions. Our results provide insights into the nature and mechanisms of mixing in RM turbulence at low Mach numbers, and yield the first measurements of key quantities in turbulence models developed to tackle these types of flows.

\section{Experiment}

The current experiments were performed in a horizontal shock tube with a square cross-section $(76.2 \mathrm{~mm} \times 76.2 \mathrm{~mm})$. During each experiment, a Mach 1.2 shock wave was created upstream of the shock tube by puncturing a diaphragm that separates the driver gas (nitrogen) from the driven gas (air). The shock wave travels down the tube (in the $x$ direction) to accelerate a density gradient created at the test section by flowing (in the vertical $z$ direction) a gravity-driven curtain of $\mathrm{SF}_{6}$ (heavy gas) that spans the test section from its top surface. The interaction of the shock wave with the air- $\mathrm{SF}_{6}$-air fluid layer deposits baroclinic vorticity, which induces the growth of perturbations at the interfaces. Since the deposition of baroclinic vorticity can occur only in the presence of a misalignment between the pressure and density fields, the nozzles through which $\mathrm{SF}_{6}$ flows into the test section were contoured to create perturbations (of primary wavelength, $\lambda=3.6 \mathrm{~mm}$ ) on either side of the curtain, which acted to modify the direction of the density gradients. After depositing the initial vorticity at the interface, the transmitted shock travels past the curtain and is reflected from the downstream end of the shock tube by a reshock wall. The reshock wave then interacts once again with the developing instability, depositing additional energy and inducing a rapid transition to turbulence (see, e.g., Hill, Pantano \& Pullin 


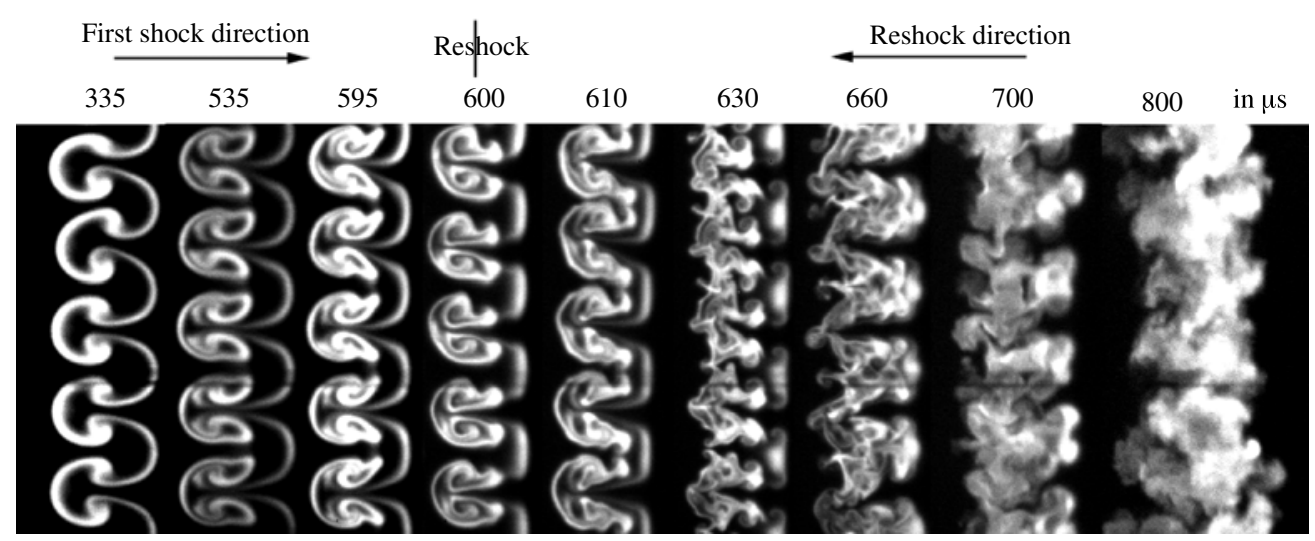

FIGURE 1. Rapid transition of the varicose RM fluid layer into a well-mixed state after reshock $(9.6 \leqslant t U / \lambda \leqslant 23.0)$.

2006; Balakumar et al. 2008), so that at this time the turbulent fluctuations are very likely three-dimensional in nature. For the present experiments, the reflecting wall has been adjusted such that the reshock wave impacts the curtain at $t=600 \mu \mathrm{s}$ (non-dimensional time, $t U / \lambda=17.2$, where $t$ is the measurement time after first shock interaction with the curtain, $U$ is the mean particle velocity behind first shock and $\lambda$ is the primary wavelength of the perturbation). All turbulence statistics are presented at $200 \mu \mathrm{s}(t U / \lambda=5.7)$ after reshock (or equivalently $800 \mu$ s after first shock; $t U / \lambda=23.0)$.

The instability is investigated by mixing the $\mathrm{SF}_{6}$ with acetone vapour and small glycol droplets from a fog generator, and using simultaneous PIV-PLIF diagnostics. A planar horizontal cross-section of the evolving fluid layer was illuminated by laser light sheets of two different wavelengths $(266 \mathrm{~nm}, 532 \mathrm{~nm})$ from frequency-multiplied $\mathrm{Nd}$ :YAG pulsed lasers. The fluorescence from the acetone was digitally registered to calculate the instantaneous density field. The density field was complemented by a simultaneous PIV velocity-field measurement that captures the streamwise $(x)$ and spanwise $(y)$ velocity components (the out-of-plane $z$-velocity component is not measured). Both the PIV and PLIF cameras were equipped with sharp cut-off filters to prevent image cross-contamination. Further experimental details are provided elsewhere, including discussions on quantitative concentration measurement with PLIF (Tomkins et al. 2008), combining PLIF with a simultaneous PIV measurement and the stability and characterization of the initial conditions (Balakumar et al. 2008), and a detailed discussion of the present flow field, including a range of velocity and density statistics with sampling errors for certain quantities (Balakumar et al. 2012).

\section{Planar density and velocity measurements}

A time series of the transition of the RM fluid layer to a turbulent state after reshock is shown in figure 1. This sequence was assembled from an ensemble of instantaneous realizations obtained from repeated runs of the same experiment with the reshock occurring at $t U / \lambda=17.2$. While the primary instability does not cause a transition and results in well-ordered structures that cause limited mixing until $t U / \lambda \sim 17.2$ after first shock, the interaction with a reshock wave creates a substantial increase in the mixing within a short duration of $t U / \lambda \leqslant 5.7$. Figure 2 shows a simultaneous PIV-PLIF field 


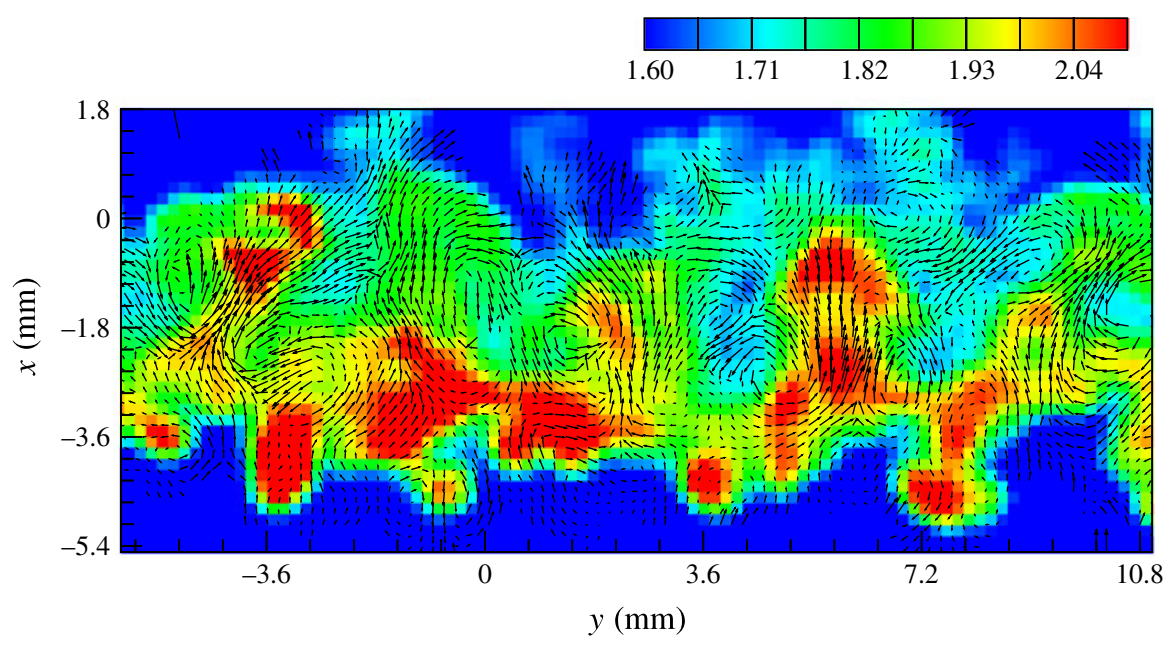

FIGURE 2. Simultaneous PIV-PLIF field showing vortical structures after reshock $(t U / \lambda=23.0)$. Contours are mixture density $\left(\mathrm{kg} \mathrm{m}^{-3}\right)$.

of the well-mixed fluid layer after reshock $(t U / \lambda=23.0)$. It is clear that the ordered fields observed earlier have disintegrated into a flow driven by a disordered distribution of vortices. Larger clumps of heavier gas occur downstream of the centreline (in the direction of the first shock) resulting in an asymmetric material distribution and enhanced mixing.

The mixing of the density field can be quantified by the density p.d.f. before and after reshock (figure 3). The p.d.f.s are calculated over a region consisting of 5.18 wavelengths in the spanwise direction and 3.36 wavelengths in the streamwise direction. Both before and after reshock, on average, the heavier fluid is converted into lower density fluid by mixing as evidenced by the lowering of the p.d.f.s at higher densities as time progresses (marked with arrows in figure 3). The loss of the heavier material is compensated by an increase in the p.d.f. of lower density material. Although the data for each of these curves was obtained from a separate realization of the experiment, the total mass of gas contained within the control volume remained within $2 \%$ between the realizations (figure 3 , inset). This implies that very little surrounding flow is actively entrained by the turbulent mixing zone beyond $\sim 1.7 \lambda$ of the centreline. At late times after reshock, a strong peak in the density p.d.f. forms at a peak $\mathrm{SF}_{6}$ concentration of $c \sim 0.08$.

\section{The density-specific volume correlation, $\langle\rho v\rangle$}

\subsection{Motivation}

While p.d.f.s provide information about the distribution of materials during the mixing process, moments of the p.d.f. provide insights beyond quantification of the mixing state. In VD flows, moments of the p.d.f. have dynamical importance to the turbulence and thus the mixing process itself. We study the mixing process here using the correlation between fluctuating specific volume and density, $b=-\langle\rho v\rangle$, also known as the DSC. Here $\rho$ and $v$ represent the density and specific volume fluctuations, respectively, and $\langle\cdot\rangle$ represents an average (typically an ensemble average). As we will discuss, $b$ is a fundamental quantity in second-moment turbulence modelling 


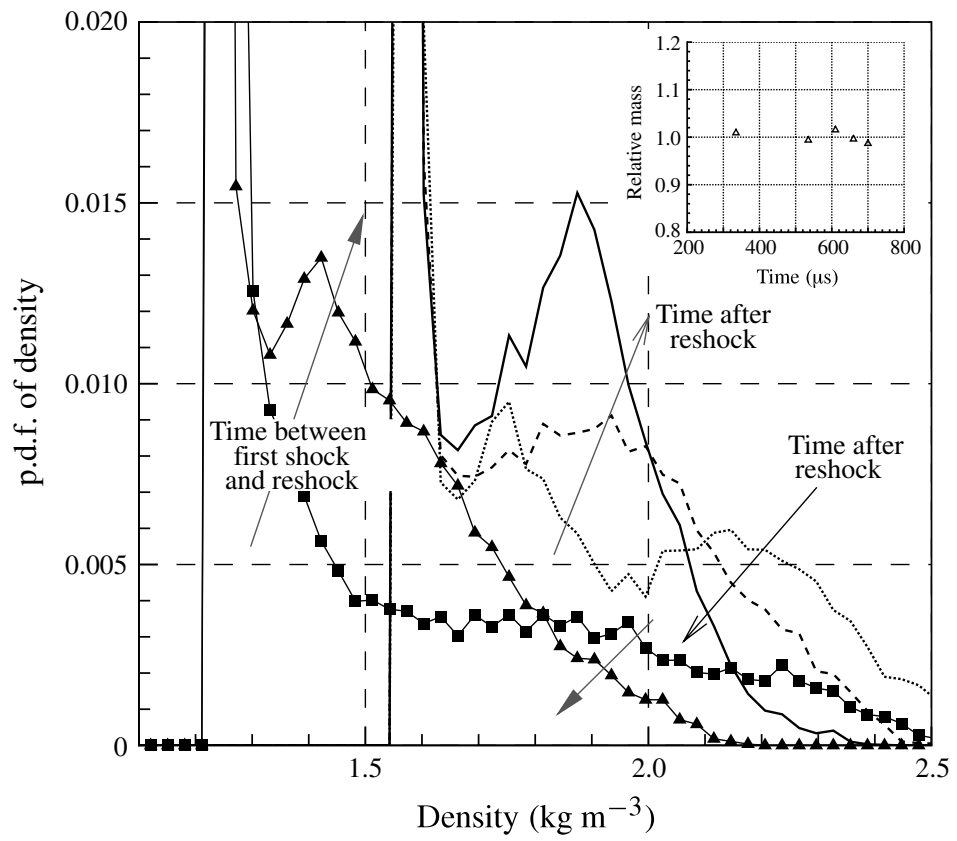

FIGURE 3. Time evolution of the p.d.f. of instantaneous density field before and after reshock: $\mathbf{\square}, t U / \lambda=9.6 ; \boldsymbol{\Delta}, t U / \lambda=15.4$; dotted line, $t U / \lambda=17.5$; dashed line, $t U / \lambda=18.9$; solid line, $t U / \lambda=20.1$.

approaches for VD flows, and through the moment equations has a direct role in the physics of the turbulence and mixing. The DSC also carries information on the instantaneous state of the mixing. The DSC is a second moment; it is, however, related to a first moment, the mean specific volume, $V$ :

$$
+\bar{\rho} V=1-\langle\rho v\rangle=1+b .
$$

By definition, $b$ is non-negative and equals 0 when the flow is fully mixed, as in this case the mean specific volume is the inverse of the mean density. As is peculiar to statistical approaches to VD flows, the mean specific volume is an independent variable. One can write an equation for the mean specific volume:

$$
\frac{\partial}{\partial t} V+\left(U_{j} V\right)_{, j}=2 V D-\left\langle u_{j} v\right\rangle_{, j}+2\langle v d\rangle
$$

where $D=U_{i, i}$ and $d=u_{i, i}$ are the mean and fluctuating dilatation, respectively. In second-moment closure approaches, $b$ plays a fundamental role in the production of turbulence (see below) to which end an equation for $b$ is typically carried,

$$
\frac{\partial}{\partial t} b+U_{j} b_{, j}=-\frac{1+b}{\bar{\rho}}\left(\bar{\rho} a_{j}\right)_{, j}-\bar{\rho}\left\langle v u_{j}\right\rangle_{, j}+2 \bar{\rho}\langle v d\rangle,
$$

which follows from (4.2). In a heuristic sense $b$ plays the role of the concentration variance in buoyantly driven flows in the Boussinesq approximation, when $\rho \ll \bar{\rho}$ (Launder 1989); in this case

$$
b \approx \frac{\left\langle\rho^{2}\right\rangle}{\bar{\rho}^{2}}
$$


which is an approximation used in some second-order closures (e.g. Gregoire, Souffland \& Gauthier 2005). As initially shown by Livescu et al. (2009) and further discussed by Schwarzkopf et al. (2011), however, using the explicit transport equation for $\langle\rho v\rangle$ as a closure for VD flows captures important physics, particularly in regimes away from the Boussinesq approximation.

The dynamical significance of $b$ follows from its role in the mass flux equation, as discussed by Livescu et al. (2009). If $a_{i}=\left\langle\rho u_{i}\right\rangle / \bar{\rho}$, then the normalized mass flux equation is written

$$
\begin{aligned}
\frac{\partial}{\partial t}\left(\bar{\rho} a_{i}\right)+\left(\bar{\rho} \tilde{U}_{j} a_{i}\right)_{, j}= & b P,_{i}+\bar{\rho}\left\langle v p_{i}\right\rangle-\bar{\rho} a_{j}\left(\tilde{U}_{i}-a_{i}\right)_{, j} \\
& +\frac{\bar{\rho}_{, j}}{\bar{\rho}}\left(\left\langle\rho u_{i} u_{j}\right\rangle-R_{i j}\right)+\bar{\rho}\left(a_{i} a_{j}\right)_{, j}-\left(\left\langle\rho u_{i} u_{j}\right\rangle_{, j}+\bar{\rho}\left\langle u_{i} d\right\rangle\right) .
\end{aligned}
$$

Thus, $b$ plays a primary role in the production of the mass flux, $b P,{ }_{i}$. Further, the primary production term of the kinetic energy of the turbulence, $k$, is $a_{j} P_{, j}$ (Livescu et al. 2009), and thus $b$ is part of the feedback between the material mixing and the turbulence that does the mixing.

\subsection{Measurement of the DSC}

Although Reynolds-averaged Navier-Stokes (RANS) models are heavily employed in the fluid mechanics and aerospace communities, detailed examinations of terms within RANS models are scarce for VD flows. Individual terms in VD transport equations such as (4.3) and (4.5) have heretofore not been measured experimentally: only high-resolution simulations have yielded estimates of terms in these types of model equations for VD flows. For example, high-resolution weighted essentially non-oscillatory (WENO) simulations have been used to examine amplification of turbulence through production of turbulence kinetic energy (TKE) after reshock (Schilling \& Latini 2010). Also, in a reshocked RM flow, Moran-Lopez \& Schilling (2013) examined mechanisms of the TKE evolution in a RANS framework, observing that shear production was balanced by molecular and turbulent diffusion of TKE as the dominant terms. An experimental estimate of the DSC was computed by Banerjee, Gore \& Andrews (2010a) in a related flow for comparison with a modified version of the Besnard et al. (1992) mix model. This estimate was based upon the Rayleigh-Taylor experimental data at low Atwood number from Banerjee, Kraft \& Andrews (2010b). Results for terms in the evolution equation of $b$ are scarcer yet, owing to the specific nature of the problem and the relatively recent derivation and presentation of these particular equations. To the best of the authors' knowledge, results here are limited to a detailed examination of direct numerical simulation data by Livescu et al. (2009). Hence, perhaps the most significant single contribution of the present work is to provide the first experimental measurements of these quantities.

To measure the DSC and investigate its evolution, an ensemble of instantaneous velocity-density fields of the developing RM curtain is assembled from repeated runs of the same experiment starting from nominally identical initial conditions. Previous RM experiments investigating transition to turbulence, despite their novelty (Rightley, Vorobieff \& Benjamin 1997; Vorobieff, Rightley \& Benjamin 1998, 1999), suffered from unstable initial conditions that led to (non-repeatable) large-scale features that had not fully dissociated into smaller vortices as expected from a turbulence cascade (see figure $2 a$ of Vorobieff et al. 1998). The present experimental facility has been upgraded (see Balakumar et al. 2008) to stabilize the initial conditions from experiment to experiment, allowing accurate ensemble averaging and Reynolds 
decomposition. Since the base flow on which the turbulent fluctuations are present is sensitive to small changes in the experimental parameters, a subset of 9 runs was selected (from a total of 115 runs) with closely controlled experimental parameters (i.e. with the following maximum variation between shots: Mach number $\leqslant \pm 0.3 \%$, structure width $\leqslant \pm 3 \%$, streamwise location jitter $\leqslant \pm 2 \%$ of structure width). From these realizations, turbulence quantities are calculated by a true ensemble average between the realizations followed by a spanwise average (where appropriate) to enhance convergence.

The variation of the DSC across the width of the curtain is shown in figure $4(a)$ for the initial condition, before reshock and after reshock. The DSC for the initial condition was calculated from a single synthetic realization with the mean density calculated as the spanwise average of Mikaelian's initial condition equations (details in Balakumar et al. 2008). The pre-reshock and post-reshock DSCs were calculated by taking a true ensemble average followed by a spanwise average. After reshock and at $t U / \lambda=23.0$, the DSC shows a double peak structure with a stronger peak downstream of the centreline. This is consistent with the higher mean density observed at the downstream side of the centre of mass and is associated with greater clumpiness of the heavy gas on the downstream side. The peak value of the DSC was measured to be $0.007 \pm 0.001$. The peaks occur on either side of the centre of mass, near the edges of the turbulent fluid layer. This is consistent with the presence of material structures (sometimes called vortex projectiles (Zabusky 1999)) that are sensitive to minor variations in the initial conditions. Thus, the variation of the density introduced by these material structures, both between instantaneous realizations and in the spanwise direction, creates large fluctuations in the ensemble and spanwise averages resulting in larger DSCs.

Note that while experiments allow the generation of ensembles, high-resolution simulations are often limited to a single realization. Therefore, DSCs were calculated from instantaneous density fields of single realizations using the spanwise-averaged density for the mean density. Using this procedure, the profiles continued to show the double-peaked structure (although the profiles were less smooth) and had a maximum peak of 0.014. The effect of the type of average on estimates of $b$ is addressed in further detail by Balakumar et al. (2012).

One expression for the evolution of the DSC in a VD turbulent flow is given by (4.3). We may also follow Besnard et al. (1992), in which the evolution equation is written as

$$
\frac{\partial b}{\partial t}+\tilde{U}_{j} b_{, j}[1]=2 a_{j} b_{, j}[2]-2 a_{j}(1+b) \frac{\bar{\rho}_{, j}}{\bar{\rho}}[3]+\bar{\rho}\left(\frac{\left\langle u_{j} \rho v\right\rangle}{\bar{\rho}}\right)_{, j}[4]+2 \bar{\rho}\langle v d\rangle[5]
$$

where $a_{j}$ represent the mass flux, $d$ represents the divergence of the velocity fluctuation and $\sim$ represents Favre-averaged quantities (Besnard et al. 1992). In this form certain terms are recast to have a more direct link to models. At late times $(t U / \lambda \geqslant 23.0)$ in the present flow, the initial periodicity of the structures in the $y$ direction has vanished due to the turbulent mixing. Hence, with the flow in a well-mixed state, and in a thin curtain, the streamwise derivatives dominate the balance equation. The Favre-averaged mean streamwise velocity was verified to be very close to its Reynoldsaveraged counterpart. Under such conditions, following a particle of fluid, the DSC evolves depending upon the relative magnitudes of terms $2-5$ in (4.6). It should be noted that the velocity fluctuations are not solenoidal and therefore term 5 could be non-negligible (Livescu et al. 2009). With the present simultaneous velocity-density 


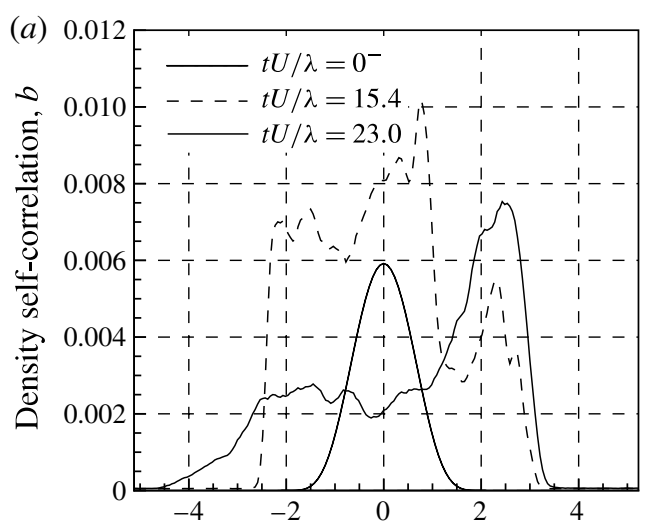

Distance from centre of mass (mm)

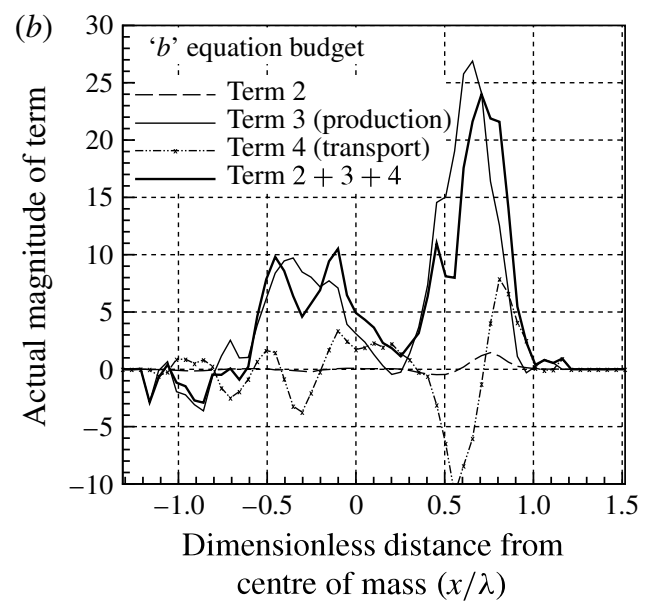

centre of mass $(x / \lambda)$

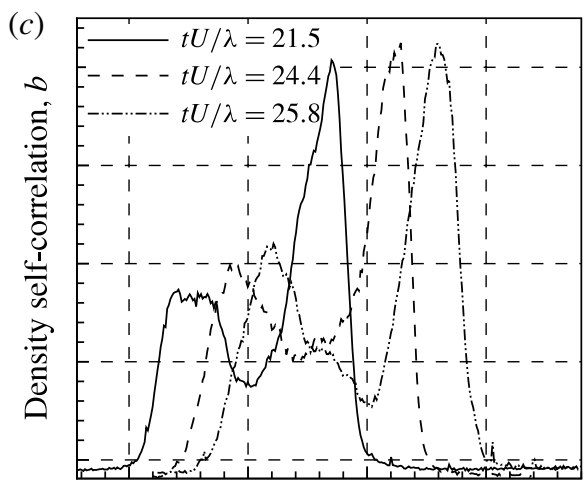

Streamwise distance

FIGURE 4. (a) Variation of the DSC parameter at three different times: before first shock $\left(t U / \lambda=0^{-}\right)$, after first shock but before reshock $(t U / \lambda=15.4)$ and after reshock $(t U / \lambda=23.0)$. (b) Experimental comparison of the relative magnitudes of the production and convection terms in the DSC evolution equation $(t U / \lambda=23.0)$. (c) Variation of DSC after reshock at three different times $(t U / \lambda=21.5,24.4,25.8)$.

data, some of these terms can be evaluated to investigate their relative importance to understand the drivers behind evolution of the DSC, and hence the RM mixing.

Figure $4(b)$ shows the variation of terms $2-4$ in (4.6). It is found that the production term (term 3) exceeds the convection term (term 2) by more than a factor of 10. The transport term (term 4) is also relatively small through most of the mixing region. Therefore, in the absence of the destruction term (term 5), the peak DSC can be expected to change by $0.0036\left(24 \times 150 \times 10^{-6}\right)$ in $150 \mu \mathrm{s}$. Similar experiments performed earlier have shown that the DSC only varies very slowly with time during this period of investigation, however (figure $4 c$ ). This slow variation is only feasible if the production term is roughly balanced by the dissipation term $(2 \bar{\rho}\langle v d\rangle)$ in the well-mixed state after reshock. Therefore, the dissipation term is inferred to have a similar magnitude to the production term but opposite in sign. This is similar to the behaviour found in Rayleigh-Taylor flows (Livescu et al. 2009). 
Hence, the measurements of individual terms in this evolution equation provide new insights into which mechanisms are crucial in the physics and modelling of the fundamental correlation $-\langle\rho v\rangle$ in a developing RM mixing flow. The mechanisms of production and dissipation are dominant, while the convection and transport terms are smaller. Further, we infer that the production is balanced by the dissipation, so in this sense the flow is in a type of equilibrium (at least with regards to the evolution of $b$ ). In a later section we explore the issue of 'time to equilibrium': some measures of how long it takes to achieve this equilibrium in the DSC. In the following section, we seek to quantify the state of development of the unstable flow that is driving the evolution of $b$, and hence the overall mixing.

\section{Probing the state of the flow}

\subsection{An inertial subrange?}

The above results provide insights into the state of the mixing using the density field, and insights into mechanisms of mixing and the evolution of $b$ using the density and velocity fields. In this section, we obtain direct insights into the state of the flow field from the velocity data, by estimating relevant turbulent length scales. In particular, we examine whether or not an inertial range exists in the current flow, which in a mixing flow also corresponds to the 'mixing transition' criteria of Dimotakis (2000).

We begin with estimates of the small scales. We estimate the TKE dissipation rate using the PIV data. Correcting for resolution effects requires an iterative approach here, because the specifics of the correction are a function of the Kolmogorov scale itself. For this mixing flow, it is appropriate to estimate the kinematic viscosity of the gaseous mixture, one expression for which is given by Youngs (1984) as $v_{\text {mix }}=\left(\mu_{1}+\mu_{2}\right) /\left(\rho_{1}+\rho_{2}\right)$. While accurate for low Atwood numbers (Banerjee et al. $2010 b$ ), for larger density ratios it is appropriate to use a more detailed expression for the dynamic viscosity of a binary mixture, as given by Reid, Prausnitz \& Sherwood (1977),

$$
\mu_{m i x}=\frac{y_{a} \mu_{a}}{y_{a}+y_{s} \phi_{a s}}+\frac{y_{s} \mu_{s}}{y_{s}+y_{a} \phi_{s a}} .
$$

Here subscript $a$ denotes air and $s$ denotes $\mathrm{SF}_{6}, y_{i}(i=a, s)$ are volume fractions and $\mu_{i}$ are the post-shock dynamic viscosities of the air and $\mathrm{SF}_{6}$. The factors $\phi$ are given by

$$
\phi_{a s}=\left[1+\left(\frac{\mu_{a}}{\mu_{s}}\right)^{1 / 2}\left(\frac{M_{s}}{M_{a}}\right)^{1 / 4}\right]^{2}\left(8\left[1+\left(\frac{M_{a}}{M_{s}}\right)\right]\right)^{-1 / 2}
$$

and

$$
\phi_{s a}=\phi_{a s}\left(\frac{\mu_{s}}{\mu_{a}}\right)\left(\frac{M_{a}}{M_{s}}\right),
$$

where $M_{i}$ are the molecular weights of the pure gases.

These expressions make clear that the mixture viscosity is a function of volume fraction, and thus changes over time and space. Unlike canonical turbulent flows, the viscosity here introduces significant variability into quantities such as the Reynolds number. For the present flow, the average volume fraction of the heavy gas within the measured curtain region is approximately $y_{s} \approx 0.2$ (Orlicz 2012), although at late time it may reduce to $y_{s} \approx 0.1$. Assuming $y_{s}=0.2$, we find a post-shock estimate 
of $\mu_{\text {mix }}=1.9 \times 10^{-5} \mathrm{~kg} \mathrm{~m}^{-1} \mathrm{~s}^{-1}$, and thus $v_{\text {mix }}=8.2 \times 10^{-6} \mathrm{~m}^{2} \mathrm{~s}^{-1}$. Note that an assumption of $y_{s}=0.1$ yields a kinematic viscosity estimate of $v_{\text {mix }}=1.1 \times 10^{-5} \mathrm{~m}^{2} / 2$, so this variation alone will lead to an increase in $R e$ of over $30 \%$. Hence, the calculations of $R e$ and associated length scales in this and related mixing flows should be viewed within the context of this degree of variability.

Using the two measured velocity components, and assuming local isotropy where necessary, we obtain an initial estimate of dissipation that neglects resolution effects, $\epsilon^{u} \approx 1.1 \times 10^{4} \mathrm{~m}^{2} \mathrm{~s}^{-3}$, where the superscript $u$ denotes an under-resolved estimate. Incorporating the nomenclature of Dimotakis (2000) for length scales, we calculate the Kolmogorov scale as

$$
\lambda_{K}=\left(v^{3} / \epsilon\right)^{1 / 4},
$$

and our initial estimate (based on the initial dissipation value) is $\lambda_{K}^{u}=$ $15 \mu \mathrm{m}$. Corrections to dissipation estimates from under-resolved PIV and hotwire measurements are discussed by Tanaka \& Eaton (2007) and Antonia, Zhu \& Kim (1993). In the present work, the vector spacing is $181 \mu \mathrm{m}$, yielding a dimensionless resolution of $\Delta x^{*}=\Delta x / \lambda_{K} \approx 12$. The error in $\epsilon$ induced by this limitation is quite severe, approximately $50 \%$ low (see Antonia et al. 1993); this is adequate for our purposes here, however, owing to the weak dependence of the Kolmogorov scale on $\epsilon$. A corrected dissipation value is thus $\epsilon \approx 2.2 \times 10^{4} \mathrm{~m}^{2} \mathrm{~s}^{-3}$, which, in turn, yields a final Kolmogorov scale of $\lambda_{K} \approx 13 \mu \mathrm{m}$ (or, to more accurately reflect the uncertainty in the estimate, $\lambda_{K} \approx O(10) \mu \mathrm{m}$ ). Comparing with $\lambda_{K}^{u}$, we also see the resolution had only a minor effect on the estimate.

Following the concepts of Dimotakis (2000), the lower end of the range (in physical, not wavenumber, space) decoupled from small and large scales is the 'inner-viscous' scale $\lambda_{v}$. With a measurement of $\lambda_{K}$ in the flow, $\lambda_{v}$ may be estimated directly using

$$
\lambda_{v} \approx 50 \lambda_{K} \approx 0.6 \mathrm{~mm} \text {. }
$$

At the other end of the spectrum, we seek a length scale decoupled from the large scales at which the turbulence is forced. With the largest scale denoted $\delta$, this decoupled 'Liepmann-Taylor' scale represents the size of a growing viscous layer based upon a $\delta$-scale sweep across the flow. It is estimated as

$$
\lambda_{L}=5.0 R e^{-1 / 2} \delta
$$

The outer scale of the flow, $\delta$, is taken to be the height $h$ of the unstable layer. In the present flow, this is measured to be $\delta=h=6.5 \mathrm{~mm}$ (Balakumar et al. 2012). A Reynolds number in vortex-driven flows may be chosen as

$$
\operatorname{Re}_{\Gamma}=\Gamma / v,
$$

where $\Gamma$ is the circulation of a representative vortex or region, which may be measured from vorticity maps computed directly from PIV data (see, e.g., Tomkins et al. 2003). In this flow, the measured circulation after reshock is $\Gamma \approx 0.1 \mathrm{~m}^{2} \mathrm{~s}^{-1}$ (Balakumar et al. 2008), leading to $R e_{\Gamma}=12,200$. These values of $\delta$ and $R e_{\Gamma}$ yield an estimated Liepmann-Taylor scale of $\lambda_{L} \approx 0.3 \mathrm{~mm}$.

A flow with an established inertial range will have $\lambda_{L} / \lambda_{v} \gg 1$, with this quantity representing the dynamic range, or scale, of the inertial range itself. In this sense, this ratio is a measure of the development of the turbulence, and indeed in recent work Lombardini, Pullin \& Meiron (2012) include this as part of a criteria for transition (here they suggest the existence of an inertial range requires at least a decade of 
scales, $\left.\lambda_{L} / \lambda_{v}>10\right)$. Our goal here is to establish an order-of-magnitude estimate of $\lambda_{L} / \lambda_{v}$. From the results above it is clear we have

$$
\lambda_{L} / \lambda_{v} \approx 1
$$

Hence, this flow is just at the point where an inertial range is being established: the size of the range is zero and growing. This is also the point at which a mixing transition would begin to occur. This is an interesting result, because in the earlier analysis of the DSC evolution equation we saw that production and dissipation were approximately in balance. These results suggest that perhaps the criterion $\lambda_{L} / \lambda_{v}>1$ is not critical for this type of equilibrium to occur in the density field, but instead $\lambda_{L} / \lambda_{v} \approx 1$ may be sufficient. These results are also broadly consistent with the findings of Lombardini et al. (2012), who find in a large eddy simulation (LES) study of a shocked single interface that transition to fully developed turbulence occurs only for $M \geqslant 1.56$, when measured by the presence of a $-5 / 3$ power law in the velocity spectra, and only for $M \geqslant 3.0$, when measured by scale separation at late times.

We may also consider the criterion developed by Zhou, Robey \& Buckingham (2003a) and Zhou et al. (2003b) for mixing transition in time-dependent flows. Equating the upper bound of the inertial range with a laminar diffusion layer, the authors propose an alternative scale $\lambda_{D}=C \times(v t)^{1 / 2}$, with $C$ varying from $\sqrt{15}$ to 5.0 depending on the flow. This is an additional criteria that must be satisfied for mixing transition, as the upper scale is taken to be $\min \left\{\lambda_{L}, \lambda_{D}\right\}$. Here, at $t=800 \mu$ s and with $C=5.0$, we obtain $\lambda_{D} \approx 0.4 \mathrm{~mm}$. Strictly speaking, this does not change the analysis, as $\min \left\{\lambda_{L}, \lambda_{D}\right\}=\lambda_{L}$; however, it is interesting to note that we have

$$
\lambda_{v} \approx \lambda_{L} \approx \lambda_{D}
$$

at this time.

In this flow, then, the time-dependent criteria is just satisfied as the inertial range is forming, and intuitively this is exactly what one would want in such a criterion. It is not clear that this result can be universal, however, as significant vorticity is added during reshock, injecting energy at scales throughout the spectrum, to more quickly transition the flow, and this effect cannot be captured by the time-dependent criterion of Zhou et al. (2003b). For example, a comparable flow without reshock is the singly shocked results of Balakumar et al. (2008), and these are clearly not fully turbulent at $t=815 \mu \mathrm{s}$; the same is true for shocked gas cylinder results at $t=1000 \mu \mathrm{s}$ (Tomkins et al. 2008).

\subsection{Length-scale estimates using local and global measures}

In the previous subsection we obtained one estimate of the inner-viscous scale $\lambda_{v}$ based upon our measurement of the Kolmogorov scale. With the simultaneous PIV-PLIF measurements, we are able to explicitly evaluate the approach in Dimotakis (2000) in which length scales (such as $\lambda_{v}$ ) are calculated as functions of large-scale observables. For many accelerated, complex flows such an evaluation is not possible due to diagnostic limitations. Developing straightforward expressions for length scales as a function of some global Reynolds number is necessary for understanding scaling with $R e$, and permits broader application of the approach, particularly with regards to complex experimental systems in which only $R e_{h}=h \dot{h} / v$ is available, e.g. in highenergy density systems (Robey et al. 2003) or challenging laboratory environments (Weber et al. 2012). The general expression for $\lambda_{v}=f(R e)$, however, is based upon results for turbulent jets, which are fundamentally different from many RM flows in 


$\begin{array}{lc}\text { Approach used to estimate } \lambda_{v} & \text { Value of } \lambda_{v}(\mathrm{~mm}) \\ \text { Local, } \lambda_{K}=f(\epsilon, v) & 0.6 \\ \text { Local, } R e=f\left(u^{\prime}, \delta\right) & 0.4 \\ \text { Global, } R e=f(\Gamma, v) & 0.3 \\ \text { Global, } R e=f(h, \dot{h}, v) & 0.3\end{array}$

TABLE 1. Estimates of $\lambda_{v}$ based upon local and global information.

certain ways, such as the presence of a mean shear. We will investigate the validity of this relation here in an unsteady, accelerated (RM) flow.

As discussed previously, $\lambda_{v}$ may be estimated directly from the dissipation and viscosity using $\lambda_{v} \approx 50 \lambda_{K}$. The more general expression of Dimotakis (2000) for the inner-viscous scale is

$$
\lambda_{v} \approx 50 R e^{-3 / 4} \delta,
$$

which can be evaluated here using both local and global estimates of Re. A local Reynolds number may be defined in terms of turbulent fluctuations, $R e_{u}=u^{\prime} \delta / v$. We estimate the velocity scale by using the width of the velocity fluctuation histograms for $u$ and $v$ in Balakumar et al. (2012), which yield an average fluctuation of $u^{\prime}=9.2 \mathrm{~m} \mathrm{~s}^{-1}$. The outer scale of the flow, $\delta$, is taken to be the height $h=6.5 \mathrm{~mm}$ of the unstable layer, as discussed previously. Our estimate of $R e$ based on local information (turbulent fluctuations) is thus $R e_{u}=7300$. Equation (5.10) is then used to compute our second estimate of $\lambda_{v} \approx 0.4 \mathrm{~mm}$.

These two length-scale estimates based on local information may then be compared with estimates based upon large-scale, global information. There are two approaches here also. Using the previously defined circulation Reynolds number, $\operatorname{Re}_{\Gamma}=\Gamma / v=$ 12200 , yields an estimate of the inner-viscous scale of $\lambda_{v}=0.3 \mathrm{~mm}$. A large-scale Reynolds number may also be calculated in a growing RT or RM layer as $R e_{h}=h \dot{h} / \nu$; as discussed above, for many systems $h$ and $\dot{h}$ may be the only experimental observables from which a Reynolds number may be calculated. Here $h$ is chosen as above, and $\dot{h}$ is measured as $13 \mathrm{~m} \mathrm{~s}^{-1}$ when averaged over the first $200 \mu \mathrm{s}$ after reshock (Balakumar et al. 2008), yielding a second estimate of a global or largescale $R e, R e_{h}=10300$. This leads to a second global-based length-scale estimate of $\lambda_{v} \approx 0.3 \mathrm{~mm}$.

The various estimates of $\lambda_{v}$ are summarized in table 1 . We see that there is variation in these four estimates by approximately a factor of two, so the different approaches, based on the Kolmogorov scale $\lambda_{K}=f(\epsilon, v)$, a local $\operatorname{Re}=f\left(u^{\prime}, \delta\right)$, a global $\operatorname{Re}=f(\Gamma, v)$ and an alternative global $\operatorname{Re}=f(\delta, \dot{h}, v)$, do yield different lengthscale estimates, but not significantly different when viewed in the context of the overall level of precision of a mixing-transition type of analysis. Hence, these results provide insights into the level of accuracy, approximately a factor of two, that one might expect in estimating length scales used in a mixing-transition analysis based solely upon large-scale observables such as $h$ and $\dot{h}$. To within this level of accuracy, the above results provide confirmation of this methodology in an RM flow.

\subsection{Time to equilibrium}

We may also use the present results to begin to explore the topic of 'time to equilibrium' in RM flows: the time it takes to move from an initial quiescent state, 
through instability and transition, to some form of equilibrium or fully developed state, which in this case is an equilibrium in the evolution equation for the DSC. One may characterize this duration in terms of the Kolmogorov time scale,

$$
\tau_{\eta}=\left(\frac{\nu}{\epsilon}\right)^{1 / 2},
$$

which based on the dissipation at the time of measurement is $\tau_{\eta} \approx 20 \mu \mathrm{s}$. The time to equilibrium expressed in terms of the Kolmogorov time scale is $t_{e q}^{*}=t / \tau_{\eta} \approx 40$. It is important to state here that these estimates cannot be strictly valid, as the notions of an equilibrium state and turbulent cascade that underpin Kolmogorov-type quantities are simply not present in these types of flows during most of the time in question. Nevertheless, the estimate presented here is one approach for quantifying the time to equilibrium, or time to the start of an inertial range, expressed in terms of concepts (such as $\tau_{\eta}$ ) that are understood and accepted by the community. While interesting in a general sense in terms of non-equilibrium, unsteady turbulence, such an estimate also yields insights into when one might expect turbulence models or simulation approaches that are based on the concept of a cascade to be applicable.

An alternative approach is to employ a time scale that reflects the large scales, such as an eddy turnover time, $\tau_{e d d y}=L / u^{\prime}$, where $L$ is the layer height and the prime denotes fluctuations associated with large-scale vortices in the flow. Here we obtain $\tau_{e d d y} \approx 700 \mu \mathrm{s}$, and so equilibrium is achieved at $t_{e q}^{*}=t / \tau_{e d d y} \approx 1$. Interestingly, equilibrium in the $b$ evolution equation is achieved in one eddy turnover time in this flow. The fact that the layer is reshocked with the reflected wave is likely a contributing factor in this (intuitively short) time to equilibrium.

\section{The Boussinesq approximation}

In buoyancy-driven flows, the Boussinesq approximation is typically used to simplify the governing equations. It is useful to investigate the validity of this approximation in RM turbulence, especially in light of the observations that: (i) the density ratio $\rho_{2} / \rho_{1}$ is 5 for the unmixed fluids here; and (ii) larger density clumps of material occur downstream of the centre of mass for the present varicose initial condition at Mach 1.2 after reshock. Figure 5 shows a comparison of the DSC $(b)$ to the first term of its Taylor expansion $\left(\left\langle\rho^{2}\right\rangle / \bar{\rho}^{2}\right)$ (Chassaing et al. 2002). Interestingly, throughout most of the flow the two values are similar, indicating that non-Boussinesq effects are not significant at the measurement time over most of the layer. In light of the VD initial condition, this result suggests that the developing turbulence and other mixing mechanisms (as discussed by Tomkins et al. 2008) have reduced VD effects in many regions as the flow evolves. Hence, the validity of the approximation will necessarily vary as a function of time, and for many flows vary as a function of position. Further, in a challenge to predictive modelling for mixing, this implies that the Boussinesq approximation is most valid when and where significant mixing has already occurred.

Despite the good agreement seen in figure 5 over most of the layer, the Boussinesq approximation is not valid everywhere in this flow field. Observable variations $(\leqslant 10 \%)$ occur near the downstream edge of the fluid layer, around the peak of the DSC. Therefore, the Boussinesq equations would slightly over-predict the mass flux near the downstream edge at late times after reshock. 


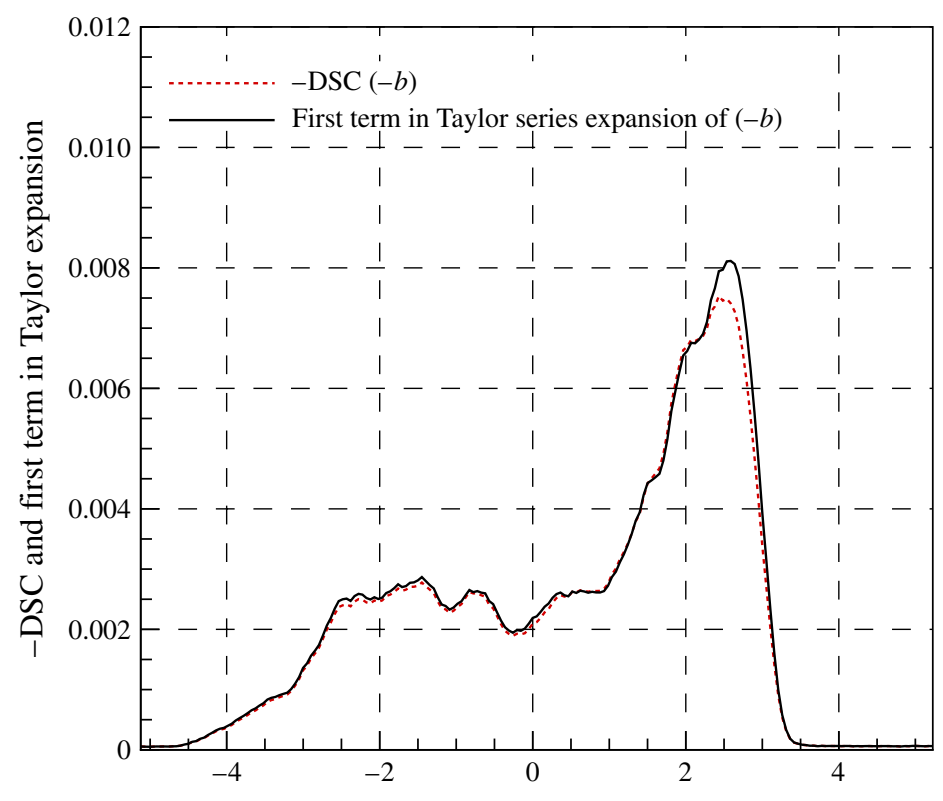

Distance from centre of mass $(\mathrm{mm})$

FIGURE 5. (Colour online) Validity of the Boussinesq approximation: comparison between DSC $(b)$ and first term in the Taylor expansion $\left(\left\langle\rho^{2}\right\rangle / \bar{\rho}^{2}\right)$ at late time after reshock $(t U / \lambda=23.0)$.

\section{Density structure functions}

Classical scaling laws for scalar turbulence have been of interest since the original works of Obukhov and Corrsin. Much work has been done exploring anomalous scaling behaviour, as evidence has accumulated that scalar fluctuations depart from classical behaviour in important ways, including a departure from isotropy at the small scales and intermittency. (For further discussion of this topic, see the detailed review by Warhaft (2000).) Nevertheless, researchers continue to probe mixing flows for evidence of universality, such as classical scaling of power spectra $(-5 / 3$ exponent) or structure functions $(2 / 3$ exponent). Several researchers have examined inhomogeneous, unsteady flows, and have found some evidence of this behaviour (Vorobieff et al. 1998) despite the departure from fundamental assumptions. Here we examine the second- and fourth-order longitudinal structure functions of density. In the present flow, we have evidence that the classical $2 / 3$ region should not exist over any significant extent, as the inertial range is only beginning to form. A plot of $\left(S_{n}=\overline{\left\langle(\rho(x+\delta x)-\rho(x))^{n}\right\rangle^{\text {Area }}}\right)$ calculated for the present data is shown in figure 6, where the overline represents an area average. The second-order structure function shows a power law scaling with a best-fit exponent of 1.23 , which is closer to a $4 / 3$ law than a $2 / 3$ law. The fourth-order structure function in the present work shows a power-law behaviour with an exponent of 2.73. It should be noted that the present density fields appear to be advanced in their mixing when compared visually to the previous work that showed the $2 / 3$ scaling (Vorobieff et al. 1998). This result is consistent, however, with the earlier analysis of turbulent length scales, finding $\lambda_{L} / \lambda_{v} \approx 1$ : while there will exist some small region of the curve to which a $2 / 3$ slope is tangent, no such region appears of any significant extent; moreover, the slope for 


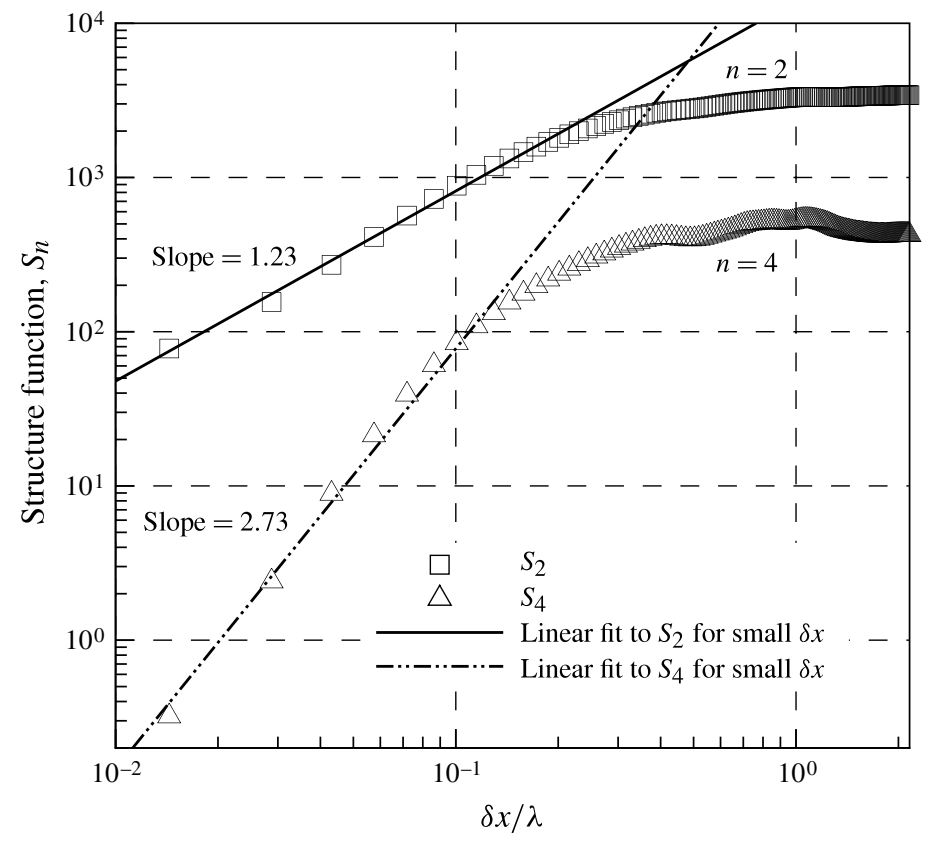

FIGURE 6. Second- and fourth-order longitudinal structure functions of the density at late time after reshock $(t U / \lambda=23.0)$.

small $\Delta x$ is clearly different. This result is also consistent with the examination of structure functions of the velocity field in a similar flow (Vorobieff et al. 2003). Here a decomposition was performed into ensemble-averaged and fluctuating components, and a 2/3-type scaling appears in the structure function of the fluctuations, but does not appear for the total (mean plus fluctuating) field at a comparable time.

It is interesting to note that for the small scales, the slope is significantly steeper than the classical result. One interpretation for the steeper slope is as follows. The present experiments consider a mixing flow in a thin-layer regime; that is, unlike most classical mixing problems, there is no fresh unmixed fluid introduced as the instabilities and mixing evolve, as discussed in Tomkins et al. (2008). In the limit of very long times in such a flow, the fluids must approach a fully mixed state, with the p.d.f. of density (or concentration) evolving to a delta function. As a result, the structure function will ultimately trend to zero for all $\delta x$, as fluctuations vanish. The behaviour shown in figure 6 is consistent with this concept, provided that the small scales are trending towards this limiting state the fastest, and thereby steepening the scaling exponent. Over time, of course, the difference at all scales would be expected to diminish in such a flow.

The fact that the structure function does not exhibit classical scaling, although unsatisfying in searches for universality, is important in our attempts to deal properly with complex transitional flows. One implication of the appearance of a universal scaling is that the emergence of a $-5 / 3$ or $2 / 3$ region would not be a sufficient condition, or even convincing evidence, of the existence of well-developed turbulence. From a modelling perspective, for example, it would not be clear that models based on fully developed turbulence assumptions are valid in regimes in which classical scaling 
of the density field is observed. In this sense, the present results preserve the role of classical scaling as a valuable statistical diagnostic for estimating the state of the flow.

\section{Conclusions}

Shock-induced turbulence in reshocked light-heavy-light fluid layers with varicose perturbations is investigated using simultaneous PIV-PLIF diagnostics to provide insights into the nature of mixing in such flows. Measurements are performed at low Mach numbers (Mach 1.2) and at late times after reshock $(t U / \lambda=23.0)$, with $\operatorname{Re}_{\Gamma}=\Gamma / \nu=12200$. The DSC function $b$, directly measured in such flows for the first time, shows a double-peaked structure after reshock. This behaviour is a characteristic feature of the flow and suggests faster mixing near the edges of the turbulent fluid layer when compared with the core.

Second-moment turbulence models in VD flows often utilize an explicit evolution equation for the DSC. Here we directly measure several terms in such an evolution equation for the first time, providing new insights for turbulence modellers. The production term in the $b$ evolution equation is observed to be much larger than the transport and convection terms (equation (4.6)) throughout most of the flow. In addition, profiles of the DSC parameter at various times after reshock show slow temporal variation when compared with the shock propagation time scale, allowing the inference that the dissipation term is similar in magnitude and opposite in sign to the production term. This is consistent with observations in Rayleigh-Taylor mixing, and indicates a type of equilibrium in $b$.

Simultaneous PIV measurements are used to probe the state of the turbulence. Calculations of various length scales to examine whether the flow is undergoing a mixing transition indicate that an inertial range is only beginning to form at this time, i.e. $\lambda_{L} / \lambda_{v} \approx 1$, despite the type of equilibrium observed in the evolution of the DSC. At Mach 1.2, this result is broadly consistent with the findings of Lombardini et al. (2012) in a closely related flow, in which transition to fully developed turbulence (roughly corresponding to $\lambda_{L} / \lambda_{v}=10$ ) was only observed for Mach $\geqslant 3$.

In many experiments, such as high-energy-density/inertial-confinement-fusion systems, calculation of these length scales from directly measured small-scale quantities is simply not possible due to diagnostic limitations, so predictions of a mixing transition must rely on large-scale observables. We investigate the validity of these types of length-scale estimates, that is, those based upon large-scale, global Reynolds numbers, in the mixing transition analysis by comparing with two estimates derived from directly measured flow quantities. Although the global expressions were derived for turbulent jets (Dimotakis 2000), these estimates are found to agree with local estimates to within a factor of two for the inner-viscous length scale, suggesting that, in at least this shock-accelerated flow, length-scale estimates based upon simple, large-scale observables are providing reasonably consistent results.

The validity of the Boussinesq approximation is examined. A density ratio of $\rho_{2} / \rho_{1}=5$ for the unmixed fluids suggests that non-Boussinesq effects may be important in this flow. While likely important at earlier times, after reshock we observe that over most of the layer the turbulence is highly Boussinesq, with the largest effects occurring near the peak of the DSC, although even these are only moderately non-Boussinesq in character $(\leqslant 10 \%)$. The observations are consistent with the idea that as an inertial subrange is established, and the turbulence drives the flow to a more mixed state, VD effects are moderated. This may not be the case, however, 
for single-interface flows in which large regions of unmixed fluid are continually introduced into the mixing zone.

We compute rough estimates for the time it takes to achieve $\lambda_{L} / \lambda_{v} \approx 1$ from an initial quiescent state. In terms of dissipative time scales, this time is $t_{e q}^{*}=t / \tau_{\eta} \approx 40$; in terms of the time scales associated with the larger motions, we observe $t_{e q}^{*}=t / \tau_{\text {eddy }} \approx 1$.

The result that the inertial range is only beginning to form suggests that the secondorder structure function should not exhibit a broad range of the classic 2/3 power-law behaviour, and indeed this is found to be the case. A power-law behaviour is observed for small scales over a range of about a decade with a steeper slope, however, which is explained in terms of the asymptotic behaviour of the structure function in a mixing flow without unmixed fluid being introduced: ultimately the function must trend to zero for all $\delta x$, and it does so most rapidly at the small scales.

Future work might include simultaneous density-velocity measurements at multiple times to probe the temporal evolution of the quantities and physics discussed here. Also, experiments with stronger shocks would yield insights into Mach number effects and permit examination of higher-Re VD turbulence.

\section{REFERENCES}

AntoniA, R., ZhU, Y. \& Kim, J. 1993 On the measurement of lateral velocity derivatives in turbulent flows. Exp. Fluids 15, 65-69.

Arnett, W. D., Bahcall, J. N., Kirshner, R. P. \& Woosley, S. E. 1987 Supernova 1987A. Annu. Rev. Astron. Astrophys. 27, 629-700.

BAKosi, J. \& Ristorcelli, J. R. 2011 Stochastic diffusion process for density-dependent multi-material turbulent mixing. Tech. Rep. LA-UR-11-01618. Los Alamos National Laboratory.

Balakumar, B. J., Orlicz, G. C., Ristorcelli, J. R., Balasubramanian, S., Prestridge, K. P. \& Tomkins, C. D. 2012 Turbulent mixing in a Richtmyer-Meshkov fluid layer after reshock: velocity and density statistics. J. Fluid Mech. 696, 67-93.

Balakumar, B. J., Orlicz, G. C., Tomkins, C. D. \& Prestridge, K. P. 2008 Simultaneous particle-image velocimetry-planar laser-induced fluorescence measurements of Richtmyer-Meshkov instability growth in a gas curtain with and without reshock. Phys. Fluids 20 (12), 124103.

Banerjee, A., Gore, R. \& Andrews, M. 2010a Development and validation of a turbulent mix model for variable-density and compressible flows. Phys. Rev. E 82, 046309.

BanerJee, A., Kraft, W. \& Andrews, M. $2010 b$ Detailed measurements of a statistically steady Rayleigh-Taylor mixing layer from small to high Atwood numbers. J. Fluid Mech. 659, 127-190.

Besnard, D., Harlow, F. H., Rauenzahn, R. M. \& Zemach, C. 1992 Turblence transport equations for variable-density turbulence and their relationship to two-field models. Tech. Rep. LA-12303-MS. Los Alamos National Laboratory.

Chassaing, P., Antonia, R. A., Anselmet, F., Joly, L. \& Sarkar, S. 2002 Variable Density Fluid Turbulence, Fluid Mechanics and its Applications, vol. 69, Kluwer.

Dimotakis, P. 2000 The mixing transition in turbulent flows. J. Fluid Mech. 409, 69-98.

Dimotakis, P. E. 2005 Turbulent mixing. Annu. Rev. Fluid Mech. 37, 329-356.

Gregoire, O., Souffland, D. \& Gauthier, S. 2005 A second-order turbulence model for gaseous mixtures induced by Richtmyer-Meshkov instability. J. Turbul. 6, N29.

Hill, D. J., Pantano, C. \& Pullin, D. I. 2006 Large-eddy simulation and multiscale modelling of a Richtmyer-Meshkov instability with reshock. J. Fluid Mech. 557, 29-61.

LAUnder, B. E. 1989 Second-moment closure: Present ... and future? Intl J. Heat Fluid Flow 10 (4), 282-300.

Lindl, J. D., McCrory, R. L. \& CAMpbell, E. M. 1992 Progress toward ignition and burn propagation in inertial confinement fusion. Phys. Today 377 (9), 32-40. 
Livescu, D. \& Ristorcelli, J. R. 2008 Variable-density mixing in buoyancy-driven turbulence. J. Fluid Mech. 605, 145-180.

Livescu, D. \& Ristorcelli, J. R. 2009 Mixing asymmetry in variable density turbulence. Adv. Turbul. XII 132 (1), 545-548.

Livescu, D., Ristorcelli, J. R., Gore, R. A., Dean, S. H., Cabot, W. H. \& Cook, A. W. 2009 High-Reynolds number Rayleigh-Taylor turbulence. J. Turbul. 10 (13), 1-32.

Lombardini, M., Pullin, D. I. \& Meiron, D. I. 2012 Transition to turbulence in shock-driven mixing: a Mach number study. J. Fluid Mech. 690, 203-226.

Moran-Lopez, J. T. \& Schilling, O. 2013 Multicomponent Reynolds-averaged Navier-Stokes simulations of reshocked Richtmyer-Meshkov instability-induced mixing. High Energy Density Phys. 9, 112-121.

ORLICZ, G. 2012 Incident shock Mach number effects on Richtmyer-Meshkov mixing with simultaneous density and velocity measurements. $\mathrm{PhD}$ thesis, University of New Mexico.

Reid, R. C., Prausnitz, J. M. \& Sherwood, T. K. 1977 The properties of gases and liquids. McGraw-Hill.

Rightley, P. M., Vorobieff, P. \& Benjamin, R. F. 1997 Evolution of a shock-accelerated thin fluid layer. Phys. Fluids 9 (6), 1770-1782.

Robey, H. F., Zhou, Y., Buckingham, A. C., Keiter, P., Remington, B. A. \& Drake, R. P. 2003 The time scale for the transition to turbulence in a high Reynolds number, accelerated flow. Phys. Plasmas 10 (3), 614-622.

SCHILLING, O. \& LATINI, M. 2010 High-order WENO simulations of three-dimensional reshocked Richtmyer-Meshkov instability to late times: dynamics, dependence on initial conditions, and comparisons to experimental data. Math. Acta Sci. 30B (2), 595-620.

Schwarzkopf, J., Livescu, D., Gore, R., Rauenzahn, R. \& Ristorcelli, J. R. 2011 Application of a second-moment closure model to mixing processes involving multicomponent miscible fluids. J. Turbul. 12 (49), 1-35.

TANAKA, T. \& EATON, J. 2007 A correction method for measuring turbulence kinetic energy dissipation rate by PIV. Exp. Fluids 42, 893-902.

Tomkins, C., Kumar, S., Orlicz, G. \& Prestridge, K. 2008 An experimental investigation of mixing mechanisms in shock-accelerated flow. J. Fluid Mech. 611, 131-150.

Tomkins, C., Prestridge, K., Rightley, P., Marr-Lyon, M., VorobiefF, P. \& Benjamin, R. 2003 A quantitative study of the interaction of two Richtmyer-Meshkov-unstable gas cylinders. Phys. Fluids 15 (4), 986-1004.

Vorobieff, P., Mohamed, N. G., Tomkins, C., Goodenough, C., Marr-Lyon, M. \& BENJAMIN, R. F. 2003 Scaling evolution in shock-induced transition to turbulence. Phys. Rev. E 68 (6), 065301.

Vorobieff, P., Rightley, P. M. \& Benjamin, R. F. 1998 Power-law spectra of incipient gas-curtain turbulence. Phys. Rev. Lett. 81 (11), 2240-2243.

Vorobieff, P., Rightley, P. M. \& Benjamin, R. F. 1999 Shock-driven gas curtain: fractal dimension evolution in transition to turbulence. Physica D 133, 469-476.

Warhaft, Z. 2000 Passive scalars in turbulent flows. Annu. Rev. Fluid Mech. 32, 203-240.

Weber, C., Haehn, N., OAKley, J., Rothamer, D. \& Bonazza, R. 2012 Turbulent mixing measurements in the Richtmyer-Meshkov instability. Phys. Fluids 24, 074105.

Williams, F. 1994 Combustion Theory. Perseus Books Publishing.

YAng, J., Kubota, T. \& Zukoski, E. E. 1993 Applications of shock-induced mixing to supersonic combustion. AIAA J. 31 (5), 854-862.

Youngs, D. L. 1984 Numerical simulation of turbulent mixing by Rayleigh-Taylor instability. Physica D 12, 32-44.

ZABUSKY, N. J. 1999 Vortex paradigm for accelerated inhomogeneous flows: visiometrics for the Rayleigh-Taylor and Richtmyer-Meshkov environments. Annu. Rev. Fluid Mech. 31, 495-536.

Zhou, Y., Robey, H. F. \& Buckingham, A. C. $2003 a$ Onset of turbulence in accelerated high-Reynolds-number flow. Phys. Rev. E 67, 056305.

Zhou, Y., Robey, H. F., Buckingham, A. C., Keiter, P., Remington, B. A. \& Drake, R. P. $2003 b$ The time scale for the transition to turbulence in a high Reynolds number, accelerated flow. Phys. Plasmas 10 (3), 614-622. 